\title{
A IMPLEMENTAÇÃO DO SIS FRONTEIRAS - PERSPECTIVAS PARA A AMPLIAÇÃO DO DIREITO À SAÚDE NA FRONTEIRA ARCO SUL
}

\author{
The implementation of the SIS Frontiers - perspectives for the expansion of the right to \\ Healthcare at the Arco Sul Border
}

Vera Maria Ribeiro Nogueira $^{1}$
Helenara Silveira Fagundes $^{2}$

\section{Resumo}

Este texto informa as ações e resultados da pesquisa A implementação do Programa SIS Fronteiras - perspectivas para a ampliação do direito à saúde aos usuários estrangeiros na Fronteira Arco Sul. Os objetivos do estudo foram avaliar a implementação e identificar as alterações ético-políticas e institucionais decorrentes desse dispositivo em regiões fronteiriças. As estratégias metodológicas escolhidas apontaram para duas dimensões: 1) o processo de implementação, detalhando a apreensão dos atores políticos acerca das negociações e dos arranjos efetivados, do nível de participação e da qualificação dos envolvidos em experiências de cooperação interfronteiras; 2) a dinâmica das diferentes experiências municipais, reconhecendo o significado e a incidência das variáveis contextuais locais e nacionais na readequação dos sistemas sanitários. Em relação aos principais resultados, pode-se afirmar que se identificam as dificuldades no campo da gestão. Embora a descentralização do sistema de saúde brasileiro tenha sido iniciada há algum tempo, são percebidos os gargalos na implantação de uma rede de serviços que contribuiria para a melhoria do atendimento à população fronteiriça. As dificuldades para programação de ações e os procedimentos para financiamento ainda não foram transpostos.

Palavras-chave: SIS-Fronteiras. Fronteiras. Sistemas Municipais de Saúde. Política de Saúde

\begin{abstract}
This paper reports on actions conducted in the realm of the study: The implementation of the SIS Frontiers Program - perspectives for the expansion of the right to healthcare for foreign users at the Arco Sul Border. The objective of the study was to evaluate the implementation and identify the ethical-political and institutional alterations that result from this program in border regions. The methodological strategies chosen point to two dimensions: 1 . The implementation process, providing details about how the political actors understand the negotiations and arrangements, and the level of participation and qualification of those involved in experiences of inter-border cooperation and; 2) the dynamic of the different municipal experiences, recognizing the significance and incidence of the local and national contextual variables in the re-adaptation of the healthcare systems. The main results of the study identify administrative difficulties. Although the decentralization of the Brazilian healthcare system was begun quite some time ago, bottlenecks are perceived in the implementation of a service network that would contribute to improving care for the population along the border. The difficulties in programming actions and procedures for financing have still not been transposed.
\end{abstract}

Keywords: SIS-Frontiers. Healthcare agreements. Borders. Municipal Healthcare Systems.

\footnotetext{
${ }^{1}$ Doutora em Enfermagem, professora da Universidade Católica de Pelotas e professora colaboradora do Programa de Pós-graduação em Serviço Social da Universidade Federal de Santa Catarina. E-mail vera.nogueira@pq.cnpq.br. Telefone-5548 9116-9776.

2 Doutora em Serviço Social, Universidade Federal de Santa Catarina. E-mail - helenarasf@hotmail.com.
}

Serv. Soc. \& Saúde, Campinas, SP v. 13, n. 2 (18) p. 245-260 jul./dez. 2014 ISSN 1676-6806 


\section{INTRODUÇÃO}

Este texto aborda as ações e os resultados da pesquisa "A implementação do Programa SIS Fronteiras", entre dezembro de 2009 e novembro de 2011. Contando com o apoio institucional do Conselho Nacional das Secretarias Municipais de Saúde CONASEMS - e financeiro do Conselho Nacional de Ciência e Tecnologia - CNPq -, teve como objeto de estudos os sistemas de saúde de municípios fronteiriços, buscando ampliar o conhecimento sobre a proteção sanitária e suas intercorrências nos limites territoriais do Brasil, entendendo ser o direito à saúde integral e universal para além das divisas nacionais, o qual deveria ser favorecido pelo SIS Fronteiras.

Os objetivos foram avaliar a implementação e identificar as alterações éticopolíticas e institucionais decorrentes da implantação de um dispositivo governamental incidente sobre a proteção sanitária em regiões fronteiriças - o Programa Sistema Integrado de Saúde nas Fronteiras - SIS Fronteiras. Foi desenvolvido junto aos sistemas locais de saúde das cidades gêmeas na linha de fronteira entre Brasil e Argentina, Paraguai e Uruguai, área geográfica designada pelo Ministério da Integração Regional como Fronteira Arco Sul (BRASIL, 2005).

No plano estritamente metodológico, algumas questões de encaminhamento foram inspiradas em estudo realizado pelo NEPP/UNICAMP, permitindo "discutir um elenco de fatores ou variáveis, classificadas dentro de algumas dimensões, que se encontra em políticas e programas que alcançam sucesso" (COSTA; SILVA; BIASOTO, 2008, p. 11).

A abordagem adotada pode ser classificada como estudo de múltiplos casos, permitindo a "descrição detalhada das experiências e levantando informações necessárias para a análise" (COSTA; SILVA; BIASOTO, 2008, p. 12). As estratégias metodológicas escolhidas apontaram para duas dimensões: 1) o processo de implementação do SIS Fronteiras, detalhando a apreensão dos gestores dos policy makers e profissionais de saúde locais acerca das negociações e dos arranjos efetivados, do nível de participação e da qualificação dos atores sociais envolvidos no processo, formulação e implantação das experiências de cooperação interfronteiras; 2) a dinâmica das diferentes experiências municipais, buscando dar resposta aos objetivos 
estabelecidos e reconhecendo o significado e a incidência das variáveis contextuais locais e nacionais na readequação dos sistemas sanitários. Os dados foram obtidos através de entrevistas, observação participante e pesquisa documental nas cidades selecionadas para o estudo. Em termos éticos, foi aprovado pelo Comitê de Ética em Pesquisa da Universidade Católica de Pelotas em reunião realizada em 16 de dezembro de 2010, ata n. $^{\circ} 10$.

Qualificando-se como descritivo, no que se refere aos resultados obtidos, o texto destaca os antecedentes da implementação dos programas, os atores políticos envolvidos, as institucionalidades sedimentadas, o patamar de resultados alcançados e os principais obstáculos identificados pelos gestores e profissionais de saúde. Em sua elaboração, privilegiaram-se as construções dos demais pesquisadores, além dos doutorandos e mestrandos envolvidos, expressando assim a produção coletiva. Nessa linha, optou-se pela não tradução de alguns itens, mantendo-os como elaborados originariamente.

Os resultados do estudo, no campo da gestão, caso utilizados pelos gestores e profissionais, poderão colaborar para fortalecer o programa em curso - SIS Fronteiras, desenvolvido pelo Ministério da Saúde, Secretarias Estaduais e Municipais de Saúde. E, ainda, aperfeiçoar os recursos financeiros alocados em municípios brasileiros de fronteira através dos protocolos derivados do Pacto pela Saúde, garantindo atenção integral também aos não nacionais, como um direito definido pelo Pacto dos Direitos Econômicos Sociais e Culturais sob a chancela da ONU e do Parlamento Brasileiro.

O mesmo ocorre com o Pacto pela Saúde, especialmente após a promulgação do Decreto Presidencial n. ${ }^{\circ}$ 7.508, que dispõe sobre a organização do Sistema Único de Saúde - SUS -, o planejamento da saúde, a assistência à saúde e a articulação interfederativa, e dá outras providências, regulamentando a Lei n. ${ }^{\circ} 8.080$ de 1990. O Decreto regula a estrutura organizativa do SUS, garantindo maior segurança jurídica para o gestor, definindo as responsabilidades das três esferas de gestão e reafirmando o papel do controle social no estabelecimento de diretrizes para o planejamento no SUS; aspectos esses já preconizados no Pacto pela Saúde. A partir desse Decreto, os serviços de saúde oferecidos devem ser organizados na Região de Saúde, em Redes de Atenção à Saúde. 
O estudo articulou pesquisadores de distintas instituições acadêmicas do sul da América Latina, parceiras nos projetos desenvolvidos sobre o tema ${ }^{3}$.

\section{ATENÇÃO EM SAÚDE EM FRONTEIRAS}

Os resultados de investigações anteriores (NOGUEIRA, 2005, 2006, 2007; GIOVANELLA et al., 2007; AGUSTINI, 2008; SILVA, 2007; PREUSS, 2007) sobre a proteção social em saúde na região da fronteira entre Brasil e demais países do Mercosul apontam, reiteradamente, o aspecto financeiro como o mais significativo obstáculo assinalado pelos gestores e profissionais de saúde para garantir o atendimento aos estrangeiros que demandam ao SUS.

Igualmente pelo lado da população brasileira usuária do sistema nacional de saúde do Brasil, a percepção do direito à saúde pelos não nacionais tem, no aspecto financeiro, o limite para esse atendimento (NOGUEIRA, 2008; PREUSS, 2007). Ou seja, no plano ético-político, há o reconhecimento do direito universal e integral à saúde, entretanto, no trânsito para a sua institucionalização como política pública, há forte restrição em decorrência da insuficiência de aportes financeiros para fazer face ao acréscimo de gastos derivados da ampliação e qualificação do atendimento.

Ressalta-se, tendo em vista a sua interferência acerca do objeto deste estudo, as distintas causas da demanda, de modo que as mais relevantes são a gratuidade do sistema e a qualidade dos serviços de saúde prestados no Brasil. A procura também é influenciada, especialmente em regiões fronteiriças empobrecidas, pela concepção integral de saúde do Sistema Único de Saúde - SUS. Uma parcela da população estrangeira acorre ao SUS como forma de aceder aos benefícios socioassistenciais, favorecidos pelo ingresso no sistema. Os Secretários Municipais da região de fronteira reiteram essas afirmações, complementando com outras dificuldades a serem enfrentadas, como a heterogeneidade do povoamento que se expressa em composição étnica, comunidades indígenas, elevado processo migratório, diferenças nas condições

\footnotetext{
${ }^{3}$ Equipe executora à época da realização do estudo - Profa. Dra. Vera Maria Ribeiro Nogueira - UCPEL/ UFSC - Coordenação; Profa. Dra. Elizabeth Cerchiaro Ortega - UDELAR - Uruguai; Profa. Dra. Helenara Silveira Fagundes - UCPEL; Profa. Dra. Lislei Teresinha Preuss - UNIJUÍ; Profa. Mte. Luisa dos Santos - UNAM - Misiones - Argentina; Profa. Dda. Maria Geusina da Silva - UNIAMÉRICA; Profa. Mte. Nora Margarida Jacquier - UNAM - Misiones - Argentina; Profa. Mte. Stella Mary Garcia Aguero - UNA - Paraguai; Profa. Mte. Carmen Garcia - Instituto Social do Mercosul/UNA - Paraguai; Prof. Mte. Jorge Centeno - UNAM - Misiones - Argentina; Profa. Dda. Celmira Bentura - UDELAR Uruguai; Prof. Mte. Elias de Souza Oliveira - UNIAMÉRICA; Consultora - Profa. Dra. Lígia Giovanella - ENSP/Fiocruz - Brasil

Serv. Soc. \& Saúde, Campinas, SP v. 13, n. 2 (18) p. 245-260 jul./dez. 2014 ISSN 1676-6806
} 
materiais de vida, de escolaridade, moradia, saneamento, trabalho e renda e contexto geográfico (CONASEMS, 2009).

$\mathrm{O}$ acréscimo assistemático e não quantificado do atendimento dificulta o planejamento das ações, penalizando sobremaneira os municípios, pois o número de habitantes é um dos critérios para os repasses financeiros do Fundo Nacional de Saúde ao Fundo Municipal de Saúde. O déficit acarretado, coberto pelo orçamento municipal, onera as Secretarias Municipais de Saúde, ampliando as dificuldades para garantir o acesso aos bens e serviços de saúde também para a população brasileira. A situação tem sido contornada por meio de estratégias, nem sempre dentro dos marcos legais.

Tais dificuldades foram reconhecidas pelo Ministério da Saúde, que, atendendo às indicações e em parceria com o Conselho Nacional dos Secretários Municipais de Saúde - CONASEMS -, formulou dois dispositivos programáticos como tentativa de solução para minimizar a situação apresentada. Um deles foi o Programa do Ministério da Saúde Sistemas Integrados de Saúde - SIS - Fronteiras, lançado, oficialmente, em setembro de 2005, na cidade de Uruguaiana. O outro dispositivo foi a inclusão, no Pacto pela Saúde, de financiamento diferenciado para o desenvolvimento de ações e serviços sanitários nos municípios de fronteira (BRASIL, 2006). Neste texto, a avaliação incidirá sobre o Programa Sistemas Integrados de Saúde - SIS - Fronteiras.

Com base no exposto, as questões que orientaram o estudo foram as seguintes: qual foi o nível de apreensão dos gestores e profissionais sobre as proposições? As iniciativas dos sistemas locais são compatíveis com as diretrizes e os objetivos das propostas? A concepção de direito à saúde aos estrangeiros foi ampliada no sentido da universalidade e integralidade? Houve alteração dos discursos dos gestores e profissionais acerca das dificuldades de atenção à saúde dos estrangeiros? Quais as repercussões das inovações sobre os sistemas de saúde dos municípios/departamentos fronteiriços? Favorecem a cooperação entre os sistemas? Quais as proposições dos gestores e policy makers para ampliar a eficácia dos programas?

\section{BUSCA DAS INFORMAÇÕES}

Considerando-se a natureza do objeto de estudo, destacam-se algumas sinalizações sobre as referências iniciais de ordem teórica utilizadas no encaminhamento das questões metodológicas e na fase analítica. 
Um marco significativo para o estudo é a própria concepção de fronteira e seus desdobramentos nos sistemas municipais de saúde e na vida cotidiana. As fronteiras, para serem entendidas em suas complexas determinações, exigem uma visão de totalidade, superando a visão tradicional, de limite territorial e área de segmentação política e social. A noção de território, formulada por Santos (2000), foi utilizada para a análise da linha da fronteira. A linha ou zona de fronteira assinala um "espaço de interação, uma paisagem específica, com espaço social transitivo, composto por diferenças oriundas da presença do limite internacional, e por fluxos e interações transfronteiriças, cuja territorialização mais evoluída é a das cidades gêmeas" (BRASIL, 2005, p. 21).

As estratégias metodológicas previstas foram confirmadas no plano operacional e apontaram para duas dimensões: 1) o processo de implementação do SIS Fronteiras, detalhando a apreensão dos gestores dos policy makers e profissionais de saúde locais acerca das negociações e dos arranjos efetivados, do nível de participação e da qualificação dos atores sociais envolvidos no processo, formulação e implantação das experiências de cooperação interfronteiras; 2) a dinâmica das diferentes experiências municipais, buscando dar resposta aos objetivos estabelecidos e reconhecendo o significado e a incidência das variáveis contextuais locais e nacionais na readequação dos sistemas sanitários.

Destaca-se ser a preocupação maior deste estudo não os resultados efetivos das inovações, o que exigiria outra abordagem metodológica, ou uma avaliação de impacto, prematura devido ao tempo de concretização dos resultados. O ponto focal foi com os processos de implementação e sua incidência na concretização das inovações ministeriais.

Os dados empíricos que forneceram subsídios para a análise foram obtidos junto aos Secretários Municipais de Saúde, três profissionais de saúde e três informantes qualificados - policy makers - em cada uma das cidades gêmeas, considerados estes como os indicados pelos profissionais de saúde como influentes nas decisões políticas, ocupando ou não posição institucional.

Os instrumentos de coleta das informações foram entrevistas (Quadro 1) focais com os sujeitos indicados anteriormente, observação participante e análise documental referida ao desenvolvimento do SIS Fronteiras. 
As cidades foram Foz do Iguaçu; Puerto Iguazu; Ciudad del Leste; Dionísio Cerqueira/Barracão; Bernardo de Irigoyen; São Borja; Santo Tomé; Itaqui; Alvear; Uruguaiana; Paso de Los Libres; Barra do Quaraí; Monte Caseros; Bella Unión; Quaraí; Artigas; Santana do Livramento; Rivera; Jaguarão; Rio Branco; Rio Grande do Sul.

Para essa etapa, dois instrumentos foram utilizados: entrevista e observação assistemática. Na coleta de informações para compor o banco de dados, utilizou-se a entrevista semiestruturada, orientada por um roteiro, contendo perguntas sobre os eixos da pesquisa.

\section{Implementação do programa SIS Fronteiras - perspectivas para a ampliação do direito à saúde aos usuários estrangeiros na fronteira arco sul}

Serão apontadas, neste item, algumas descobertas mais instigantes e intrigantes da realidade fronteiriça, sinalizando para o alcance dos objetivos propostos. A diversidade de situações sobre a história, a trajetória e o nível de apreensão dos gestores demarca um dos impasses relativos à implementação das políticas de saúde no Brasil.

Os municípios maiores, com capacidade de planejamento mais aprimorada, indicam um bom conhecimento do programa e de sua construção ascendente, ou seja, o mesmo surge de uma necessidade local e obtêm consenso até a formulação de política pública. Inclusive se constatam referências de alguns gestores e profissionais de saúde que expressam a sua participação na construção do programa, anteriormente à sua formalização pelo Ministério da Saúde.

Contrariamente, municípios de menor porte, com uma rotatividade de profissionais muito alta, sinalizam que, até o momento, não têm conhecimento mais aprofundado do Programa, indicando ser um programa vertical e com escasso debate com os municípios. Nesses casos, atribuem o fato à ausência de oportunidades de capacitação para o projeto.

As referências desses municípios sobre a concepção e o conteúdo do programa vinculam-se à expansão do teto financeiro para atendimento dos estrangeiros devido à localização na fronteira. Mencionam a realização de um diagnóstico local, mas não se referem ao uso dele para elaboração dos Planos Municipais de Saúde nem as possibilidades do início de cooperação transfronteiriça, conforme os objetivos originários do projeto ou a sua relação com processos de avaliação e monitoramento. 
Quadro 1 - Quadro das entrevistas e qualificação dos entrevistados ${ }^{4}$

\begin{tabular}{|c|c|c|c|}
\hline & Local & Instituição & Situação funcional \\
\hline 1. & Bella Unión & Hospital de Bella União & Médico - Adjunto Hospital \\
\hline 2. & Paso de Los Libres & Secretaria de Salud Municipal & Enfermeira \\
\hline 3. & Paso de Los Libres & Secretaria de Salud Municipal & Assistente Social \\
\hline 4. & Rivera & Ministério de Saúde & Diretora de Rede de Atenção Básica \\
\hline 5. & Bernardo Yrigoyen & Hospital de Bernardo Yrigoyen & Agente de saúde \\
\hline 6. & Dionísio Cerqueira & Secretaria de Desenvolvimento Social & Assistente Social \\
\hline 7. & Dionísio Cerqueira & Secretaria Municipal de Saúde & Enfermeira \\
\hline 8. & Dionísio Cerqueira & $\begin{array}{l}\text { Secretaria Municipal de Saúde - CAP I - } \\
\text { Tri-fronteira }\end{array}$ & Psicóloga \\
\hline 9. & Dionísio Cerqueira & \begin{tabular}{|llll}
$\begin{array}{l}\text { Secretaria Municipal } \\
\text { Vigilância Sanitária }\end{array}$ & de Saúde & & - \\
\end{tabular} & Assistente Social \\
\hline 10. & Dionísio Cerqueira & Secretaria Municipal de Saúde & Secretário de Saúde \\
\hline 11. & Jaguarão & Secretaria Municipal de Saúde & Enfermeira \\
\hline 12. & Jaguarão & Secretaria Municipal de Saúde & Secretária Municipal de Saúde \\
\hline 13. & São Borja & $\begin{array}{l}\text { Vigilância Epidemiologia e Centro de } \\
\text { Processamento de Dados }\end{array}$ & Enfermeira \\
\hline 14. & Jaguarão & Secretaria Municipal de Saúde & Fisioterapeuta/Secretaria Municipal de Saúde \\
\hline 15. & Jaguarão & Secretaria Municipal de Saúde & Enfermeira \\
\hline 16. & São Borja & $\begin{array}{l}\text { Secretaria Municipal de Saúde - Diretor de } \\
\text { Planejamento, Orçamento e Projetos }\end{array}$ & Biólogo \\
\hline 17. & Itaqui & Hospital São Patrício & Administrador \\
\hline 18. & Itaqui & $\begin{array}{l}\text { Secretaria Municipal de Saúde - } \\
\text { Coordenadora do Centro de Atenção } \\
\text { Psicossocial-CAPS }\end{array}$ & Psicóloga \\
\hline 19. & Itaqui & Secretaria Municipal de Saúde & Enfermeira - Secretária Substituta \\
\hline 20. & Itaqui & Secretaria Municipal de Saúde & Enfermeira/Centro de Saúde \\
\hline 21. & Itaqui & Secretaria Municipal de Saúde - PSF & Enfermeira \\
\hline 22. & Uruguaiana & $\begin{array}{l}\text { Secretaria Municipal de Saúde - Chefe da } \\
\text { Vigilância Sanitária }\end{array}$ & Farmacêutica Bioquímica \\
\hline 23. & Uruguaiana & Secretaria Municipal de Saúde/DST/AIDS & Psicóloga \\
\hline 24. & Uruguaiana & $\begin{array}{l}\text { Secretaria Municipal de Saúde/Setor } \\
\text { Financeiro-contábil }\end{array}$ & Contador \\
\hline 25. & Uruguaiana & Secretaria Municipal de Saúde & $\begin{array}{l}\text { Administrador/Secretário de Saúde/Vice- } \\
\text { Prefeito }\end{array}$ \\
\hline 26. & Barra do Quarai & Secretaria Municipal de Saúde & Auxiliar de enfermagem/Secretária substituta \\
\hline 27. & Barra do Quarai & Secretaria Municipal de Saúde & Secretário Executivo do Gabinete do Pronasc \\
\hline 28. & Barra do Quarai & Secretaria Municipal de Saúde & Coordenador de Enfermagem \\
\hline 29. & Quarai & Secretaria Municipal de Saúde & Secretário de Saúde \\
\hline 30. & Quarai & Secretaria Municipal de Saúde & Coordenador de Vigilância Sanitária \\
\hline 31. & Quarai & Secretaria Municipal de Saúde & Auxiliar administrativo \\
\hline 32. & \begin{tabular}{|l} 
Santana do \\
Livramento
\end{tabular} & Secretaria Municipal de Saúde & Secretário de Saúde \\
\hline 33. & \begin{tabular}{|l} 
Santana \\
Livramento
\end{tabular} & Secretaria Municipal de Saúde & Coordenadora da Rede Básica \\
\hline 34. & \begin{tabular}{|ll} 
Santana do \\
Livramento
\end{tabular} & Secretaria Municipal de Saúde & Informática/Assessoria Secretário Municipal \\
\hline 35. & Barracão & $\begin{array}{l}\text { Secretaria Municipal de Saúde - Unidade } \\
\text { Básica de Saúde }\end{array}$ & Enfermeira SMS \\
\hline 36. & Foz do Iguaçu & Conselho Municipal da Saúde & Administrador \\
\hline 37. & Foz do Iguaçu & Secretaria Municipal de Saúde & Secretário de Saúde \\
\hline
\end{tabular}

Elaborado pelos autores - 2013

${ }^{4} \mathrm{O}$ nome dos entrevistados não foi indicado por razões de confidencialidade, e os relatos na íntegra estão em poder da pesquisadora responsável.

Serv. Soc. \& Saúde, Campinas, SP v. 13, n. 2 (18) p. 245-260 jul./dez. 2014 ISSN 1676-6806 
Aparentemente perceberam como uma ação do Ministério parcelar, pontual e não integrada ao programa municipal. Outros entrevistados dos municípios de menor porte indicam que o programa veio para complementar as ações municipais, desvinculadas de qualquer outra perspectiva de fronteira.

Quanto à história do programa, além dos gestores que participaram mais ativamente de sua construção e do seu lançamento, ocorrido em Uruguaiana, as indicações são sucintas e referem ser uma iniciativa do governo federal. O foco da iniciativa não foi percebido, e um dos entrevistados relatou ser voltado para a saúde da mulher, de modo que não atende a demanda do seu município, a qual é transporte para pacientes.

A trajetória da experiência não foi unívoca e expressa o comprometimento maior ou menor dos gestores e profissionais da saúde com o Programa. Há referências constantes da pesquisa realizada para construção do diagnóstico nacional sobre a saúde na fronteira e do envolvimento das Universidades Federais nesse processo, embora grande parte dos entrevistados tenha se desligado do programa após a etapa inicial.

As motivações iniciais dos gestores para adesão ao programa são referidas sempre para complementar a ação, de nível básico ou média complexidade. Nesse quesito, a percepção dos profissionais distancia-se da dos gestores. Entre os profissionais entrevistados nos municípios de médio porte, há referências da possibilidade derivada do objetivo do SIS Fronteiras de ser um instrumento que realmente levaria a uma cooperação entre os sistemas municipais além da fronteira brasileira.

Os informes autorizam afirmar o alto investimento na realização do diagnóstico inicial dos municípios fronteiriços, com expressivo envolvimento dos gestores e profissionais, além de assumirem parte dos trâmites burocráticos.

\section{SIS Fronteiras - Implementação}

Quanto ao processo de implementação do SIS Fronteiras, a partir dos depoimentos dos gestores, identifica-se um procedimento comum entre todos, ou seja, a orientação para se elaborar um diagnóstico, denominada como a primeira etapa do programa. 
A partir dessa consigna e da elaboração do diagnóstico original, a continuidade da implementação foi sendo diversificada. Uma das dificuldades identificadas nessa etapa do programa foi a alteração dos postos de comando das Secretarias Municipais de Saúde, que, devido à inexistência de um sistema de planejamento e monitoramento contínuos e de um quadro de funcionários efetivos, desfavoreceu as continuidades técnico-administrativas, perdendo-se os objetivos originais, que também não foram perseguidos pelas instâncias superiores.

Dois municípios, Quaraí e Santana do Livramento, contam com uma equipe para coordenar e encaminhar o SIS Fronteiras, conforme indicação dos gestores, de forma que ambos estão na segunda etapa do Programa.

Em termos da implementação, podem ser elencados os seguintes achados, respondendo ao objetivo sobre esse tópico.

- Presença do Conselho Municipal de Saúde para aprovação da implementação do Programa no município.

- Referências reiteradas pelos gestores da ausência de assessoria do governo federal e estadual sobre o programa.

- Ações realizadas de forma isolada pelos municípios, não se identificando iniciativas de integração transfronteiriça nem mesmo entre municípios brasileiros.

- Troca constante de pessoal responsável pelo programa, sendo que foi adquirindo, ao longo dos anos, um aspecto essencialmente burocrático e vinculado ao setor financeiro dos municípios, especialmente na fase três.

- Inexistência de processos de monitoramento e avaliação.

- A operacionalização do Programa tem sido, inúmeras vezes, reiterada pelos entrevistados e conduzida por pessoal administrativo de nível médio.

- Diversidade de programas e serviços implementados, que atendem as necessidades unicamente do sistema municipal de saúde.

- As ações programáticas do SIS Fronteiras, na percepção dos profissionais de municípios de pequeno porte, estabeleceu-se como uma alimentação de um banco de dados, sem o objetivo original.

- A partir da terceira fase, que ocorreu em 2010, não houve manifestação do Ministério sobre o andamento do Programa. 


\section{SIS Fronteiras - Resultados}

As percepções dos gestores foram analisadas tendo como referência os objetivos centrais do SIS Fronteiras, e pode-se afirmar que há um relativo consenso sobre os resultados, os quais ficaram aquém do que originariamente prometia o projeto inicial.

As falas dos gestores mais comprometidos com a qualidade da atenção e com a universalização da saúde tem clareza e indicam a necessidade de expansão do SIS Fronteiras para além dos espaços nacionais, dando conta das particularidades fronteiriças, e não unicamente transpondo responsabilidades aos gestores sem o devido respaldo institucional e financeiro. Há também indicação da escassa contribuição do programa para a qualificação da gestão municipal da saúde, pois esses aspectos não foram devidamente trabalhados pelas autoridades ministeriais.

Esses mesmos gestores apontam que o programa deveria contemplar todos os municípios de fronteira, tanto o lado brasileiro como o uruguaio.

Além disso, os profissionais têm uma visão mais acurada que os gestores e apontam aspectos significativos para a análise. Sinalizam que um dos entraves foi a mudança do objetivo de um momento para o outro, desfazendo rapidamente toda a expectativa em termos de estratégias e planos de ação, desacreditando até mesmo as expectativas menos otimistas em relação ao Programa. Foi relatada ainda a dificuldade devido às distinções nas políticas de saúde, desde aspectos mais éticos até programáticos essenciais, como o calendário de vacinação.

Os gestores e profissionais não mencionam as iniciativas que vem ocorrendo por parte do Uruguai sobre integração transfronteiriça, não se referem aos Seminários realizados nos últimos dois anos.

As falas dos gestores permitem sintetizar alguns aspectos relacionados aos objetivos previstos:

- escassa contribuição do Programa quanto aos seus objetivos originais;

- reconhecimento da importância do Programa para os municípios fronteiriços, caso seguisse as orientações programáticas iniciais e houvesse um assessoramento mais eficiente dos órgãos superiores;

- viabilidade de articular ações transfronteiriças;

- contribuição do Programa para os diagnósticos dos municípios;

- contribuição para a gestão nos municípios de pequeno porte. 


\section{BALANÇO DA EXPERIÊNCIA - ÊXITOS E FRUSTAÇÕES}

Em relação ao SIS Fronteiras, percebem-se as dificuldades no campo da gestão. Embora a descentralização do sistema de saúde brasileiro tenha sido iniciada há algum tempo, identificam-se os gargalos na implantação de uma rede de serviços que contribuiria para a melhoria do atendimento à população, sendo que as dificuldades para programação de ações ainda não foram transpostas.

Sobre a implementação do SIS Fronteiras, os objetivos iniciais do Programa não foram atingidos, e os recursos financeiros foram utilizados unicamente para ampliar a rede de serviços locais e manter atualizado o banco de dados. Os aspectos de integração, ou mesmo de cooperação transfronteiriça, previstos, igualmente não se concretizaram; nem mesmo se identificaram quaisquer iniciativas nesse sentido.

O papel dos gestores se revelou crucial e preocupante, pois a descontinuidade administrativa é constante, não permitindo que programas implementados alcancem os objetivos previstos. Uma das justificativas apresentadas para essa descontinuidade foi a ausência de assessoria do governo federal e estadual sobre o programa.

Um destaque foi a participação do Conselho Municipal de Saúde para aprovação da implementação do Programa nos municípios, com exceção de unicamente dois municípios que não informaram o papel do Conselho.

A troca constante de pessoal responsável pelo programa conferiu a ele um aspecto essencialmente burocrático e vinculado ao setor financeiro dos municípios, especialmente na fase três.

Ainda sobre a implementação e operacionalização do SIS Fronteiras, identificou-se a transformação gradativa de ações programáticas para atividades burocráticas administrativas, como alimentação do banco de dados e compras para os programas em execução, desenvolvidos a partir do diagnóstico inicial realizado. Possivelmente, devido a esse fato, a operacionalização é realizada por pessoal de nível médio. Um destaque é o município de Quaraí, que conta com uma equipe constituída de pessoal de nível superior para coordenação do Programa.

A referência ao diagnóstico inicial foi uma constante, o que autoriza a afirmar a competência do Ministério nessa primeira fase do Programa. Profissionais e gestores reconhecem que a alteração da equipe ministerial responsável pelo Programa foi um dos fatores que contribuíram para a não continuidade de acordo com a proposta original. A 
partir da terceira fase não há indicação clara da continuidade do Programa, levando a uma indefinição que desfavorece quaisquer ações programáticas.

Há o reconhecimento da importância do SIS Fronteiras para os municípios, visto que a exigência de ações cooperativas é uma constante. $\mathrm{O}$ fato de qualquer iniciativa ficar subordinada ao governo federal, visto ser área de fronteira, é um empecilho, levando a iniciativas nem sempre ao amparo da lei.

Observou-se que os recursos do SIS Fronteiras foram utilizados em uma série de programas e serviços, atendendo às necessidades do sistema municipal de saúde. A abertura para atendimento aos estrangeiros permanece a critério do gestor, mantendo-se a limitação do direito à saúde na maior parte dos municípios.

Constatou-se que as negociações e os arranjos são efetivados apenas em âmbito local, e o nível de participação e a qualificação dos atores sociais envolvidos no processo de pactuação são extremamente limitados. A segmentação das ações administrativas, favorecida pela reduzida capacidade de planejamento dos municípios e concepção de política pública, não contribui para o êxito das inovações ministeriais.

Para os estrangeiros, em relação aos serviços de saúde, a situação é ainda mais preocupante. Para a população que ali vive, muitas vezes, o acesso à saúde significa ultrapassar a fronteira: o outro lado da rua, a ponte, ou a balsa. As cidades fronteiriças nos países vizinhos, em sua maioria, não possuem atividade econômica rentável e podem ser considerados de pequeno e médio porte, carentes em infraestrutura.

As informações dos gestores e profissionais estrangeiros a respeito do SIS Fronteiras confirmam a distância entre o pretendido e o alcançado em termos de operacionalização. Ou seja, as ações não foram desenvolvidas de modo a fomentar iniciativas transfronteiriças. A cooperação existente entre Santana do Livramento/Rivera e Barracão/Bernardo de Yrigoyen antecede aos Programas implementados. Quanto à relevância e ao significado das proposições, há unanimidade em afirmar serem estratégias poderosas para a necessária cooperação entre os sistemas.

O estudo aponta para a importância de pesquisas desenvolvidas, como a qual se está vinculada, e a continuidade destas, de pensar novas questões relacionadas ao processo de pactuação em outros territórios brasileiro. Pode ajudar também a se pensar novos caminhos e estratégias para a gestão dos serviços de saúde, a descentralização e a regionalização. 


\section{Referências Bibliográficas}

AGUSTINI, J. A descentralização da política nacional de saúde e sua institucionalidade nos sistemas municipais na linha da fronteira Mercosul. 2008. 231 p. Dissertação (Mestrado em Serviço Social) - Universidade Federal de Santa Catarina, Florianópolis, 2008. Disponível em: <http://repositorio.ufsc.br/xmlui/bitstream/handle/123456789/92172/251135.pdf?seque nce $=1 \&$ isAllowed $=\mathrm{y}>$. Acesso em: 10 jul. 2008.

BRASIL. Ministério da Integração Regional. Secretaria de Programas Regionais. Programa de Desenvolvimento da Faixa de Fronteira. Proposta de reestruturação do programa de desenvolvimento da faixa de fronteira: bases para uma política integrada de desenvolvimento regional para a faixa de fronteira. Brasília: Ministério da Integração Regional, 2005.

BRASIL. Ministério da Saúde. Pactos pela saúde. [2006]. Disponível em: $<$ http://portal.saude.gov.br/portal/saude/profissional/area.cfm?id_area=1021>. Acesso em: 12 jul. 2008.

CONASEMS. Relatório executivo do Painel Saúde nas Fronteiras e o Processo de Integração Regional. In: CONGRESSO DO CONSELHO NACIONAL DE SECRETARIAS MUNICIPAIS DE SAÚDE - CONASEMS, 25. 2009, Brasília, DF. Anais eletrônicos... Brasília, DF: CONASEMS, 2009.

COSTA, V. L. C.; SILVA, P. L. B.; BIASOTO, G. (Org.). Efetividade das Políticas de Saúde: experiências bem-sucedidas na América Latina. São Paulo: Biruta, 2008.

GIOVANELLA, L. et al. Saúde nas fronteiras: acesso e demandas de estrangeiros e brasileiros não residentes ao SUS nas cidades de fronteira com países do Mercosul na perspectiva dos secretários municipais de saúde. Cadernos de Saúde Pública, Rio de Janeiro, v. 23, supl. 2, p. S251-S266, 2007. Disponível em: <http://www.scielo.br/pdf/csp/v23s2/13.pdf>. Acesso em: 16 mar. 2009.

NOGUEIRA, V. M. R.; DAL PRA, K. R; FERMIANO, S. A diversidade ética e política na garantia e fruição do direito à saúde nos municípios brasileiros da linha da fronteira do Mercosul. Cadernos de Saúde Pública, Rio de Janeiro, v. 23, supl. 2, p. S227-S236, 2007. Disponível em: <http://www.scielo.br/pdf/csp/v23s2/11.pdf>. Acesso em: 16 mar. 2009.

NOGUEIRA, V. M. R.; DAL PRÁ, K. Mercosul: expressões das desigualdades em saúde na linha da fronteira. Ser Social, Brasília, v. 1, n. 18, p. 159-188, jan.-jun. 2006. 
Disponível em: <http://periodicos.unb.br/index.php/SER_Social/article/view/186/969>. Acesso em: 20 ago. 2009.

NOGUEIRA, V. M. R. Fronteira Mercosul: um estudo sobre o direito à saúde. Florianópolis: Departamento de Serviço Social/UFSC, 2005. Relatório final de pesquisa.

NOGUEIRA, V. M. R. A implementação do programa SIS fronteiras e do Pacto pela Saúde - perspectivas para a ampliação do direito à saúde aos usuários estrangeiros na fronteira arco sul. Pelotas: Programa de Pós-Graduação em Política Social/UCPEL, 2011. Relatório final de pesquisa.

PREUSS, L. T. O direito à saúde na fronteira: duas versões sobre o mesmo tema. 2007. 172 p. Dissertação (Mestrado em Serviço Social) - Universidade Federal de Santa Catarina, Florianópolis, $2007 . \quad$ Disponível em: <https://repositorio.ufsc.br/bitstream/handle/123456789/90418/245170.pdf?sequence=1 >. Acesso em: $1^{\circ}$ abr. 2009.

SANTOS, M. O espaço do cidadão. 5. ed. São Paulo: Studio Nobel, 2000.

SILVA, M. G. da. O local e o global na atenção das necessidades de saúde dos brasiguaios: análise da intervenção profissional do assistente social em Foz do Iguaçu. 2006. 176 p. Dissertação (Mestrado em Serviço Social) - Universidade Federal de Santa Catarina, $\quad 2006 . \quad$ Dlorianópolis, em: <https://repositorio.ufsc.br/bitstream/handle/123456789/88602/236617.pdf?sequence=1 >. Acesso em: 10 out. 2009. 
